\title{
Multiple nerve root metastasis of breast carcinoma: a report of two cases
}

\author{
Jing Zhang ${ }^{1}$, Yun-Na Yang ${ }^{2}$, Cang Liu ${ }^{1}$, Yi-Peng Dong ${ }^{1}$, Yan-Lin Zhang ${ }^{1}$ \\ ${ }^{1}$ Department of Neurosurgery, Beijing Friendship Hospital, Capital Medical University, Beijing, China; ${ }^{2}$ Department of Neurosurgery, Beijing \\ Chaoyang Hospital, Capital Medical University, Beijing, China \\ Correspondence to: Jing Zhang. Department of Neurosurgery, Beijing Friendship Hospital, Capital Medical University, No.95 Yong'an Road, Xicheng \\ District, Beijing 100050, China. Email: 13910178021@139.com.
}

\begin{abstract}
Nerve root metastases are extremely rare with only a handful of cases ever reported. Metastasis to sites other than the primary site is common in malignant tumors whereas spinal ganglion metastasis is extremely rare and has been only reported in individual cases. The lumbar spine tends to be more common areas of presentation whereas breast cancer metastasis has been rarely reported. We herein reported two cases of breast carcinoma metastasis to multiple spinal nerve roots. The metastasis sites were S1 nerve root in Case 1 and left L5 and bilateral cervical nerve roots in Case 2. On magnetic resonance imaging (MRI), the nerve roots in the intervertebral foramen zones appeared thickened and contrast-enhanced MRI exhibited intense enhancement. Pathological examination showed that these primary lesions were breast cancer in both cases, and there were intracranial multiple metastases in both cases, including preoperative metastasis to multiple nerve roots (lumbar and cervical) and postoperative recurrence. The clinical course was characterized by worsening radicular symptoms - especially intractable pain. The radiologic appearance might mimic a neurogenic tumor, which is performed intervertebral foraminal area lesion, and the corresponding ganglion/ nerve root became thickened and was enhanced significantly. Surgical intervention with tumor debulking followed by radiotherapy provides local tumor control and palliation from pain, but it is palliative. Therefore, for patients with radiological manifestations of radiculopathy, the possibility of metastatic tumors should be considered.
\end{abstract}

Keywords! Breast carcinoma; case reports; magnetic resonance imaging (MRI); nerve root metastasis

Submitted Sep 09, 2020. Accepted for publication Dec 29, 2020.

doi: 10.21037 /gs-20-708

View this article at: http://dx.doi.org/10.21037/gs-20-708

\section{Introduction}

Metastasis to sites other than the primary site is common in malignant tumors. The organs, bones and lymph nodes tend to be more common areas of presentation, whereas spinal ganglion metastasis is extremely rare. To the best of our knowledge, spinal ganglion metastases have been only reported in individual cases. The lumbar spine tends to be more common areas of presentation whereas breast cancer metastasis has been reported only once (1). Nerve root metastases are rare, and the literature is full of individual cases, with no large number of cases reported. A review of the current literature revealed only 15 cases of metastases to the spinal nerve root ganglia; arising from distant solid tumors, the primary lesions in those cases were pulmonary adenocarcinoma in 3 cases (2-4), renal cell carcinoma (RCC) in 3 cases $(5,6)$, colonic adenocarcinoma in 2 cases $(2,7)$, uterine adenocarcinoma in 1 case (8), a ductal breast carcinoma (1), a Ewing's sarcoma (9), a gastro-intestinal stromal tumor (10), a follicular thyroid carcinoma (11), a cervical cancer (12), and a cervical breast-cancer (13). Herein, we reported a patient with S1 nerve root and a patient with L5 bilateral cervical nerve root metastasis of breast adenocarcinoma. One of our cases is the first reported case of metastasis to a sacral nerve root from breast adenocarcinoma, and the other is the first reported case of 

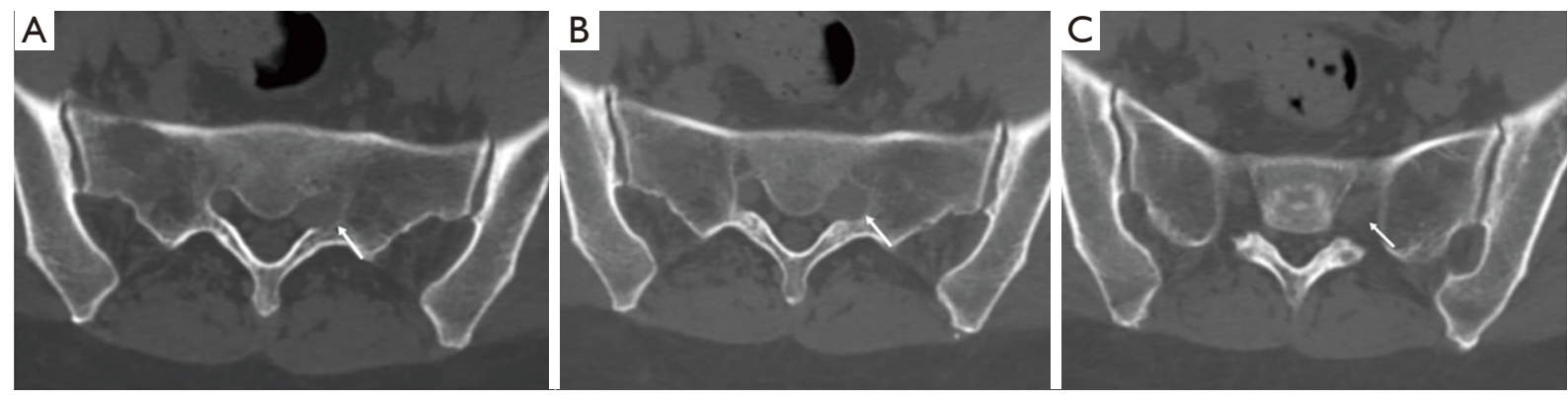

Figure 1 Preoperative magnetic resonance imaging (MRI) reveals thickening of the S1 nerve root (arrow) and enhancement of the lesion $(\mathrm{A}, \mathrm{B}, \mathrm{C})$.

multiple metastasis to lumbar and cervical nerves.

We present the following cases in accordance with the CARE reporting checklist (available at http://dx.doi. org/10.21037/gs-20-708).

\section{Case presentation}

Case 1: A 50-year-old female patient was admitted with severe intense radial pain on the left lower limb 6 years later. The pain was not alleviated by oral analgesics. Preoperative magnetic resonance imaging (MRI) revealed thickening of the S1 nerve root and enhancement of the lesion (Figure 1). Through the posterior semi-lamina approach, the intervertebral foramen was opened, and thickened ganglia and nerve roots were observed. The outer membrane was cut open to expose the tumor. The symptoms were completely relieved postoperatively and did not reappear during the follow-up period. Histologic examination confirmed the tumor to be a metastasis of breast cancer. The procedure was followed by radiotherapy. However, the patient succumbed to intracranial multiple metastases 29 months later.

Case 2: A 54-year-old woman who underwent breast carcinoma surgery 14 years ago was admitted to our department with postoperative pain and numbness on the left leg, and was investigated for cancer recurrence. On MRI, the left L5 and bilateral cervical nerve roots appeared thickened and enhancement was obvious after injection of contrast enhancer (Figure 2). The operation was performed on the waist via by the mid-posterior approach. Through minimally invasive surgery, the intervertebral foramen was opened, and the nerve ganglia and nerve roots were found to be thickened. The tumor was completely removed during the operation, and the nerve ganglia and nerve roots were well protected. Histologic examination confirmed that the tumor was a metastasis of breast cancer as determined by immunohistological staining. The procedure was followed by radiochemotherapy. However, new metastasis to the intra-spinal canal on thoracic, multiple intracranial metastases and recurrence in the L5 nerve root surgical site appeared 24 months later (Figure 3).

All procedures performed in studies involving human participants were in accordance with the ethical standards of the institutional and/or national research committee(s) and with the Helsinki Declaration (as revised in 2013). The study received ethics approval from the Ethic Commission of the Beijing Friendship Hospital, Capital Medical University (No. CMU-20-039). Written informed consent was obtained from the patients and their healthy siblings.

\section{Discussion}

Nerve root metastases manifest on CT/MRI as intervertebral foramen lesions and thickening of the involved nerve root with contrast enhancement $(1,4,5,11)$, which was also observed in our cases. Radiological findings of nerve root metastases may mimic those of neurogenic tumors, which most commonly are peripheral nerve sheath tumors (1). Clinically, nerve root metastases can mimic a radiculopathy and/or cauda equina syndrome, and it is difficult to make a definite diagnosis. According to Keen et al. 2016 (11), worsening radicular symptoms are the presenting manifestations in $90-95 \%$ of patients, which was present in our patients, who first developed typical radicular symptoms of lower limb pain and numbness. Therefore, for tumor patients with nerve root lesions and imaging findings of the above manifestations, the possibility of metastatic tumor should be considered. 

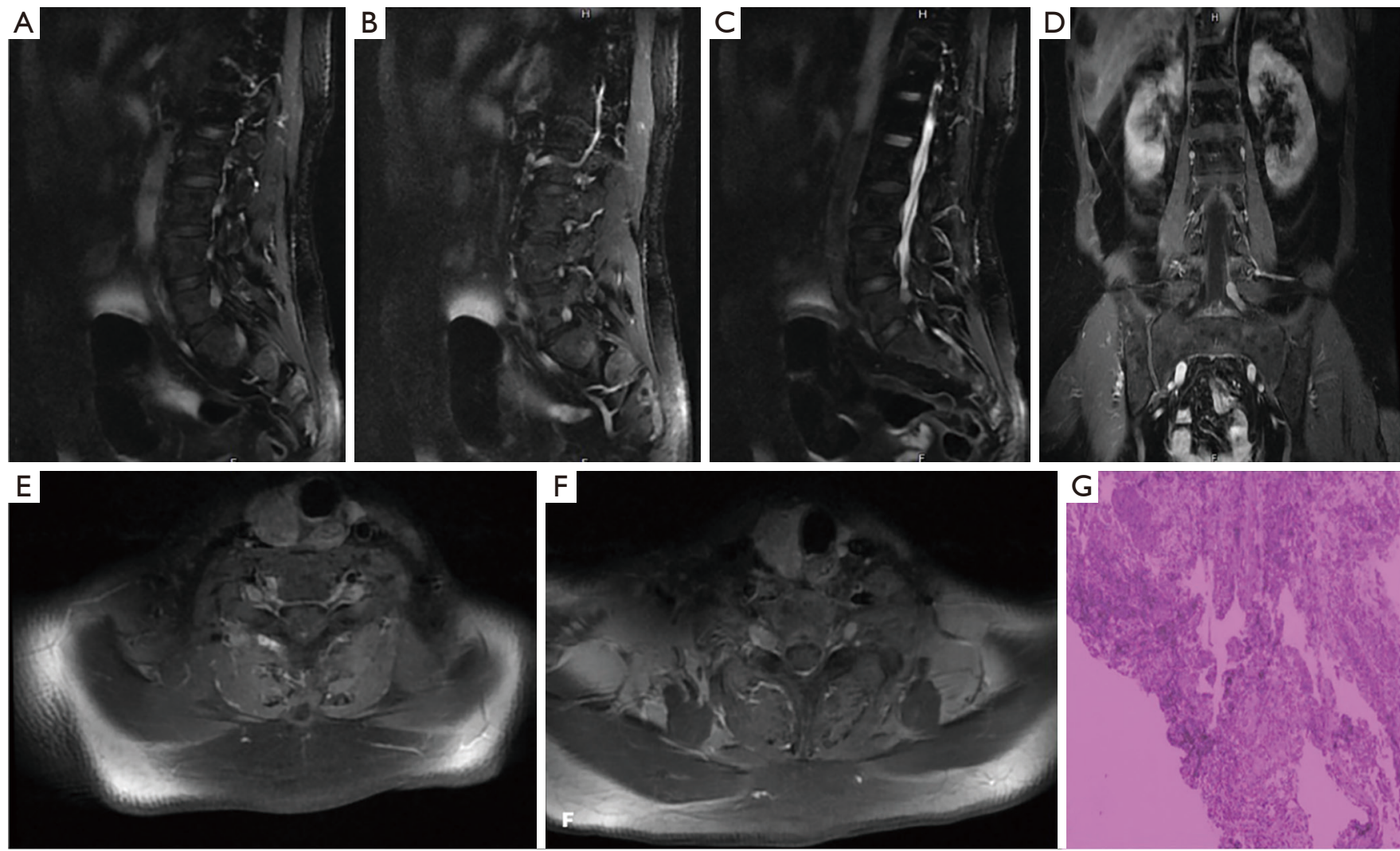

Figure 2 Preoperative T2 sagittal (A, B and C) and (D) MRI shows left L5 nerve root thickening and avid enhancement. Preoperative T2 axial MR Imaging (E, F) shows bilateral cervical nerve root thickening. Histopathological examination stained with hematoxylin and eosin confirms a metastasis of breast adenocarcinoma $(\mathrm{G}, \times 40)$.

In the literature review, except for the autopsy findings of 2 cases, one of the remaining 13 cases was treated with radiotherapy (13). One patient underwent puncture biopsy (10), which was confirmed to be metastatic tumor by pathology, and the other 11 cases were treated with surgery, and 7 of them were completely removed. Postoperative chemoradiotherapy was applied.

Both of our patients accepted surgical treatment, one underwent total resection of the tumor, which recurred 24 months after surgery, and the other underwent partial resection and decompression of the nerve root in order to avoid injury due to multiple nerve fibers traversing the tumor. The symptoms of the two patients were significantly improved postoperatively. The purpose of the operation was to alleviate symptoms and improve patients' quality of life, and make a definite diagnosis. One patient had a posterior approach to the hemilamina, and another had a minimally invasive approach to the subchannel. The operative time was not long (145 minutes in case 1 , and 170 minutes in case 2), and the blood loss was $200 \mathrm{~mL}$ in case 1 and $50 \mathrm{~mL}$ in case 2 .

Both of our patients received postoperative radiotherapy and chemotherapy while both had multiple intracranial metastases. One patient succumbed to multiple intracranial metastases 29 months later, and the other is still undergoing chemotherapy. Both patients had common characteristic of breast cancer, and at the same time had multiple intracranial metastases, including preoperative nerve root multiple metastasis (lumbar and cervical) and postoperative recurrence, suggesting cerebrospinal fluid spread. Tumor cells may be present in the cerebrospinal fluid for such patients. For such patients, literature review shows that surgery can significantly improve symptoms and quality of life. Surgery is relatively minimally invasive. Even if the tumor cannot be completely resected, nerve decompression can still be very good to improve symptoms. Accordingly, surgical treatment should be pursued actively for this kind of patients with patient consent and family 

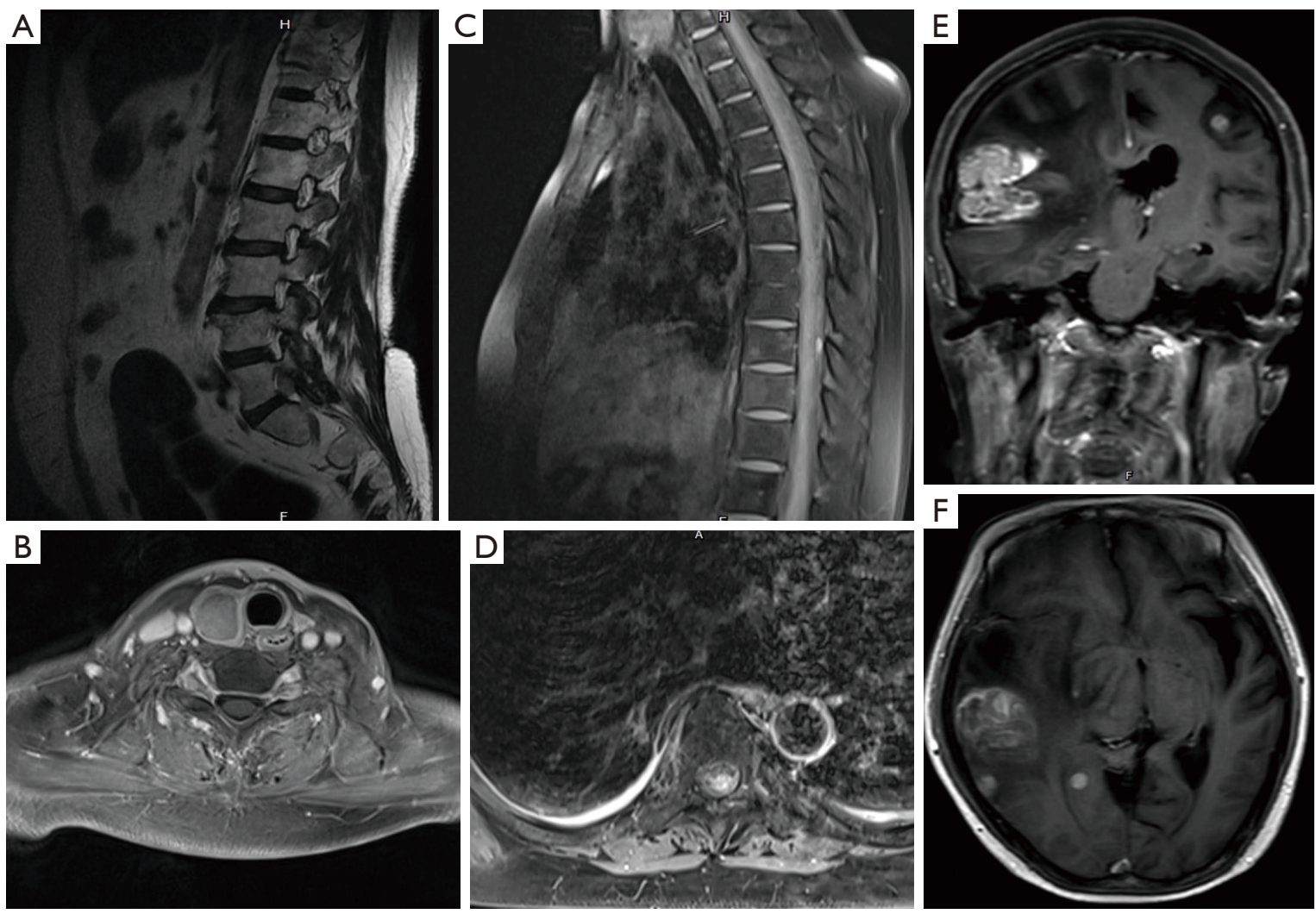

Figure 3 T2 sagittal and axial MRI shows recurrence in the L5 nerve root (A), no significant lesion in the cervical nerve roots (B), new metastasis to the intraspinal canal on the thoracic spine $(\mathrm{C}, \mathrm{D})$, and multiple intracranial metastases $(\mathrm{E}, \mathrm{F})$.

member agreement. The value of surgery lies in improving symptoms and quality of life, clarifying diagnosis and providing reference for follow-up treatment. Intraoperative neuroelectrophysiological monitoring can be applied to protect the nerve and resect the tumor as completely as possible. Both of our patients were complete disappearance of postoperative symptoms, which is consistent with the literature.

However, there are some limitations in our study. Multiple intracranial metastases occurred in both cases, among which multiple intraspinal metastases occurred in one case. There were also intracranial metastases in related literature, but there was limited literature and the followup data were incomplete. In order to delay the spread of the cerebrospinal fluid, it should be considered that intrathecal chemotherapy or chemotherapy in the Ommaya sac in the postoperative course. Local radiotherapy was the usual postoperative course, and whole brain and/or spinal cord radiation therapy should be considered using a multidisciplinary approach.

\section{Conclusions}

Although very rare, tumor metastasis to the spinal nerve root ganglion has been reported several times. The clinical course is characterized by worsening radicular symptomsespecially intractable pain. The radiologic appearance may mimic a neurogenic tumor, which is performed intervertebral foraminal area lesion, corresponding ganglion/nerve root thickened and enhanced significantly. Surgical intervention with tumor debulking followed by radiotherapy provides local tumor control and palliation from pain, but it is palliative. the possibility of metastatic tumor should be considered for tumor patients with imaging findings of nerve root lesions.

\section{Acknowledgments}

The authors express gratitude to all the technical and clinical staffs involved in patient management and laboratory diagnosis. 
Funding: None.

\section{Footnote}

Reporting Checklist: The authors have completed the CARE reporting checklist. Available at http://dx.doi.org/10.21037/ gs-20-708

Conflicts of Interest: All authors have completed the ICMJE uniform disclosure form (available at http://dx.doi. org/10.21037/gs-20-708). The authors have no conflicts of interest to declare.

Ethical Statement: The authors are accountable for all aspects of the work in ensuring that questions related to the accuracy or integrity of any part of the work are appropriately investigated and resolved. All procedures performed in studies involving human participants were in accordance with the ethical standards of the institutional and/or national research committee(s) and with the Helsinki Declaration (as revised in 2013). The study received ethics approval from the Ethic Commission of the Beijing Friendship Hospital, Capital Medical University (No. CMU-20-039). Written informed consent was obtained from the patient and her healthy siblings for the publication.

Open Access Statement: This is an Open Access article distributed in accordance with the Creative Commons Attribution-NonCommercial-NoDerivs 4.0 International License (CC BY-NC-ND 4.0), which permits the noncommercial replication and distribution of the article with the strict proviso that no changes or edits are made and the original work is properly cited (including links to both the formal publication through the relevant DOI and the license). See: https://creativecommons.org/licenses/by-nc-nd/4.0/.

\section{References}

1. Schulz M, Lamont D, Muthu T, et al. Metastasis of Breast Cancer to a Lumbar Spinal Nerve Root Ganglion. Spine

Cite this article as: Zhang J, Yang YN, Liu C, Dong YP, Zhang YL. Multiple nerve root metastasis of breast carcinoma: a report of two cases. Gland Surg 2021;10(4):1542-1546. doi: 10.21037/gs20-708
(Phila Pa 1976) 2009;34:E735-9.

2. Johnson, P.C., Hematogenous metastases of carcinoma to dorsal root ganglia. Acta Neuropathol 1977;38:171-2.

3. Oktay K, Guzel E, Bitiren M, et al. Lung Adenocarcinoma Metastasis Mimicking Peripheral Nerve Sheath Tumor: Case Report and Review of Literature. World Neurosurg 2018;120:490-4.

4. Slotty PJ, Cornelius JF, Schneiderhan TM, et al. Pulmonary adenocarcinoma metastasis to a dorsal root ganglion: a case report and review of the literature. J Med Case Rep 2013;7:212.

5. Cabrilo I, Burkhardt K, Schaller K, et al. Renal carcinoma relapse presenting as a peripheral nerve sheath tumor: A case report and brief review of the literature. Neurochirurgie 2013;59:128-32.

6. Strong C, Yanamadala V, Khanna A, et al. Surgical treatment options and management strategies of metastatic renal cell carcinoma to the lumbar spinal nerve roots. J Clin Neurosci 2013;20:1546-9.

7. Wigfield CC, Hilton DA, Coleman MG, et al. Metastatic adenocarcinoma masquerading as a solitary nerve sheath tumour. Br J Neurosurg 2003;17:459-61.

8. Uchida K, Kobayashi S, Yayama T, et al. Metastatic involvement of sacral nerve roots from uterine carcinoma: a case report. Spine J 2008;8:849-52.

9. Mitchell BD, Fox BD, Viswanathan A, et al. Ewing sarcoma mimicking a peripheral nerve sheath tumor. J Clin Neurosci 2010;17:1317-9.

10. Di Sibio A, Romano L, Giuliani A, et al. Nerve root metastasis of gastric adenocarcinoma: A case report and review of the literature. Int J Surg Case Rep 2019;61:9-13.

11. Keen J, Milosavljevic E, Hanna G, et al. Rare Intradural Cervical Nerve Root Metastasis of Follicular Thyroid Carcinoma. Cureus 2016;8:e898.

12. Li L, Wu Y, Hu L, et al. Metastatic nerve root tumor: A case report and literature review. Mol Clin Oncol 2016;4:1039-40.

13. Boese CK, Lechler P, Bredow J, et al. Atypical presentation of a cervical breast-cancer metastasis mimicking a dumbbell-shaped neurinoma. Int J Surg Case Rep 2014;5:689-93. 